\title{
A Study of Today's A.I. through Chatbots and Rediscovery of Machine Intelligence
}

\author{
Anirudh Khanna ${ }^{1}$, Bishwajeet Pandey ${ }^{1}$, Kushagra Vashishta ${ }^{1}$, Kartik Kalia ${ }^{1}$, Bhale \\ Pradeepkumar and Teerath Das \\ ${ }^{1}$ Chitkara University, Punjab, India \\ anirudhkhanna.cse@gmail.com,bishwajeet.pandey@chitkara.edu.in, \\ kushagravashishta@gmail.com,kartikalia4@gmail.com \\ ${ }^{2}$ IIITM, Gwalior, India \\ bhalepradeepkumar.iiit@gmail.com \\ ${ }^{3}$ Gran Sasoo Science Institute, Italy \\ teerath.sitani@gmail.com
}

\begin{abstract}
Artificial Intelligence in machines is a very challenging discussion. It involves the creation of machines which can simulate intelligence. This paper discusses some of the current trends and practices in AI and subsequently offers alternative theory for improvement in some of today's prominent and widely accepted postulates. For this, focus on the structuring and functioning of a simple A.I. system - chatbots (or chatter bots) is made. The paper shows how current approach towards A.I. is not adequate and offers a new theory that discusses machine intelligence, throwing light to the future of intelligent systems.
\end{abstract}

Keywords: artificial intelligence, machine intelligence, chatbots, theory of intelligent machines, Turing test

\section{Introduction}

The creation and analysis of intelligent agents (software and machines) is called Artificial Intelligence, or AI. It can be implemented in nearly each and every sphere of work. Intelligent machines can do many tasks - from labor work to sophisticated operations. Prominent trends in this field are human brain simulation, natural-language processing and neural networking etc. One of the typical examples of an AI system is a "chatbot". A chatbot is a computer program which responds like an intelligent entity when conversed with. The conversation may be through text or voice. Any chatbot program understands one or more human languages by Natural Language Processing. Due to this, the system interprets human language input using information fed to it. A chatbot may also perform some productive functions like calculations, setting-up remainders or alarms etc. A popular example of chatbot is the ALICE Bot (Artificial Linguistic Internet Computer Entity), which utilizes AIML pattern matching techniques [1]. "Turing test" is one of the most popular measures of intelligence of such systems. According to it, if a panel of human beings conversing with an unknown entity (via keyboard, for example) believes that that entity is human while the entity is actually a computer, then the computer is said to have passed the Turing test. This test was proposed by British mathematician Alan Turing in his 1950 paper titled "Computing Machinery and Intelligence" published in Mind [2]. All these developments have been made, but are we actually constantly progressing towards the higher goals of AI? These days, the evolution in AI has been limited around some key points like the fed-in information database, imitation of human abilities, targeting the Turing test and techniques of deception. Lesser novel visions are being introduced in this field and further research is going on in existing 
theories only. Also, today's AI systems "pretend to act like" intelligent entities instead of being one. For example, today's rule-based systems just follow a large set of if-else based logic (the database) while actually intelligent entities don't always do so, but apply reasoning in every stage. Thus, it is not the case that Artificial Intelligence is failing, but it has slowed down in attaining more laurels. There is a need to view AI systems from a new perspective and a novel theory of machine intelligence is the required tool for this.

\section{Functioning of Simple AI Systems}

Chatbots are one of the most elementary and popular examples of intelligent Human Computer Interaction (HCI). For realizing the strengths and weaknesses of current AI systems, we created two chatbot programs - FUTURE (in $\mathrm{C}++$ ) and SARANG (in AIML). Moreover, the SARANG Bot is hosted on the internet and can be accessed from worldwide [3]. $\mathrm{C}++$ is a typical general-purpose object-oriented programming language for creating applications. AIML (Artificial Intelligence Markup Language) is a dialect of XML which is designed especially for natural language processing agents, like the chatbot programs. Choosing AIML gave us the advantage of using the open-source ALICE AIML set directly, consisting of the 50,000 responses of ALICE chatbot in our project and made it more efficient for research [4]. This section gives a review of their structuring and functioning. AI designing generally requires the system to be differentiated into simple sections of work. In chatbots, there is a separate section that manages the transfer and understanding of messages between human user and the AI system, another section that stores the useful key-points during the chat that may be required later-on, another department that handles the errors and so on. The mutual interactions of these modules are shown pictorially in Figure 1. The program is logically divided into the following modules.

\subsection{Introductory Screen}

The welcome screen introduces the user to the program and may ask him some of his details like the name for further conversation purposes. A key point like the user name is good to be stored in a data-file with use of Data Handling.

\subsection{The Chat Interface and Manager}

This is the main region, where all the text-inputs are managed and the program fetches the best-matching answers from its database. It has to search and display from tens of thousands of rules stored in the database.

\subsection{The Database}

This is the brain of a chatbot. Database consists of an organized, manually created list of suitable replies given for possible questions that may come up from user's end. A vast range of questions has to be covered by an efficient chatbot for standing firm in the industry and it should also have more than one answer to the same kind of question to avoid redundancy of replies. Each reply should be semantically connected.

\subsection{Productivity Application}

The chatbot becomes much better when some productivity applications like a simple calculator or a dictionary or even a game is embedded in it. We introduced a calculator in the FUTURE bot. If the user types in for a calculator requirement, there is a simple calculator in the program itself. The user can work upon basic arithmetic operations, trigonometric functions and differentiate simple expressions too. These days, AI agents have much more to offer than a calculator. They can make memos, notes, set alarms and open up programs on your computers or mobiles on demand. 


\subsection{Ambiguity Handling}

Sometimes, the end-user poses questions that a program's database does not have answers to. Here, ambiguity handling comes into play. It gives answers when no relevant data is available. Its contents fit into wide range of contexts and hence, user is satisfied even for an unexpected question.

\subsection{Data Handling}

The user may not like to tell his name each time he uses the chatbot. Important data about user can be stored permanently via data file handling. We store data for future reference, and for modifying our answers such that they seem suitable for the user specifically. This makes the program more intelligent.

\subsection{Error Handling}

One example of this is seen if the user tries to divide any number by zero while using the embedded calculator application in the chatbot. The program should be capable of handling such general errors and try to continue operating within its environment even after that.

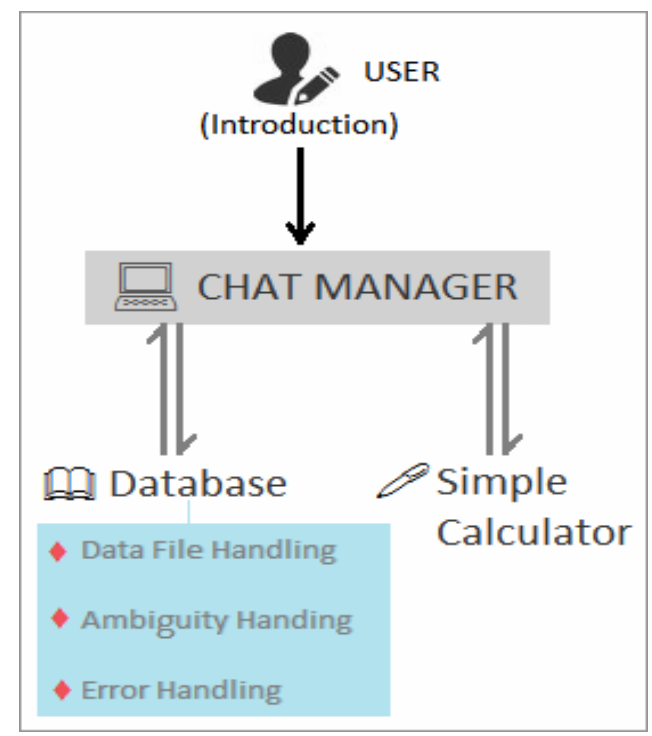

Figure 1. Interaction between the Various Modules

We infer that using AIML with designated application in AI field boosted the total speed of development of the project. It significantly increased the efficiency of the program created as it offers a lot of AI-oriented features. With well over 60,000 responses fed into it, the SARANG Bot was tested for about 1500 queries. It could manage to give suitable replies to about 1200 of them, which gives its accuracy to be $80 \%$ that has been shown in Figure 2. 


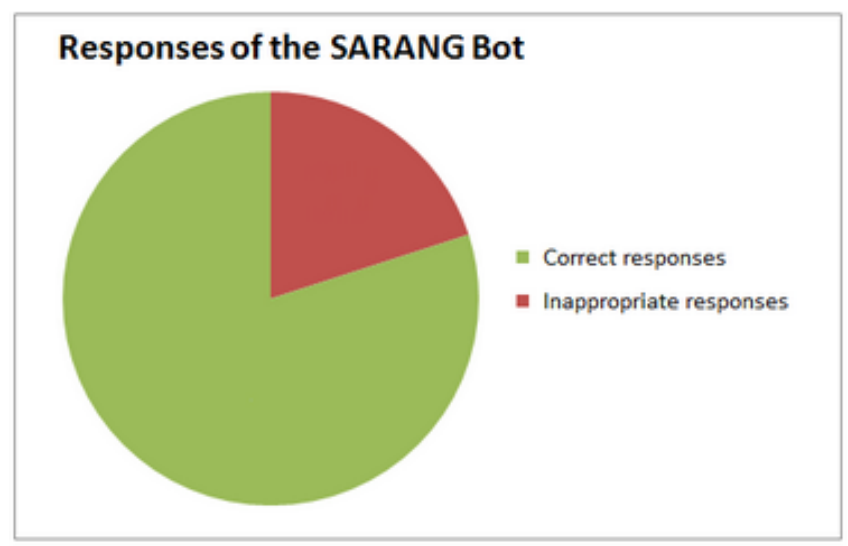

Figure 2. Performance of SARANG Bot

This is a little glimpse of how efficient and diverse AI systems can be constructed today, and that too, with not much difficulty. There are a lot of great chatbots in the world. Many have won the Loebner prize and are really very good programs. But even after all these advanced, aren't we just standing there only? The core problems with chatbot programs (and AI systems in general) remain the same. They do not hold the power to learn, hold memories of people and incidents. They do not have (or simulate) senses. They are not creative and original, just a fixed set of instructions. They do not actually understand what is going on (the conversation, for instance). They give responses by searching and finding matches from a whole lot of database inputs, that sometimes goes up to millions of patterns. [5]

\section{The Turing Test and its Problems}

The Turing test is not a perfect measure to tell if a machine is intelligent. There is perhaps no fool-proof way to tell if an entity is intelligent or not. Sometimes, unintelligent behavior is witnessed in intelligent beings. On the other hand, dumb machines may at times perform a lot of tasks more intelligently and accurately than intelligent human beings. But the Turing test has its own challenges discussed as follows.

\subsection{Limitedness}

The test only features intelligence in terms of conversational skills of a machine. Intelligence is a lot more than that.

\subsection{Short Purview}

The scope of this test is only bounded within the limits of human ability to comprehend and respond in a human language. But it is not the only intelligent behavior seen in the world. What about intelligence in animals? Also, ability to pass the Turing test is not the only sign of intelligence shown by humans.

\subsection{Unproductive Developments}

Trying to pass the Turing Test is merely a distraction from more fruitful research.

\subsection{Disillusionment of Goals}

Creating lifelike simulations of human beings is a critical task in itself. Such entities may be interesting in a game or maybe as a friendlier user interface, but they are not part of the core science of creating intelligent machines, that is, machines that solve problems 
using intelligence. The test is not compulsory to be passed for achieving higher goals of AI research.

\section{Back to the Basics: The Meaning of Intelligence}

If the present postulates need to be improved, we have to provide some alternatives. For this, we restart from the basics. There is need to rethink about the fundamental abilities upon which intelligence around us works. These are as follows.

\subsection{Arithmetic}

The power to compute is the fundamental of intelligence. It includes arithmetic operations like addition, subtraction, division and so on. Today's machines perform well on this part. They can carry out even complex calculations in no time.

\subsection{Comparison, Logic and Reasoning}

The scope of machine intelligence becomes wider when a system acquires the ability to apply logic and make comparisons. Today's computers can perform logical operations well with the principles of Boolean algebra.

\subsection{Learning, Heuristics and Memory}

Power to recollect past incidents, learn new things and gaining experience constantly are major features of intelligent entities. R\&D is going on in this direction these days. Heuristics is being implemented in newer software and as time passes, the ability of machines to learn and gain experience will grow.

\subsection{Senses}

This is how we know about the environment around us. We humans are fortunate enough to have really efficient and effective set of senses. Some animals are said to have even greater abilities to sense. A machine with rightly installed equipments to sense the surroundings will prove to be a great body for its intelligent brain. But as of now, even a caterpillar can outperform machines in the ability to know about their surrounding environment.

\subsection{Perception}

This is how we receive and process the signals our senses provide us. This is how creativity is born to intelligence. To have a machine with a perception seems a distant dream of AI.

\subsection{Consciousness}

Consciousness of intelligent beings is perhaps the most difficult property to define. It is even more difficult to be implemented in a machine. How can an abiotic system come to notice the presence of itself in the world? This is a question which is really difficult to answer. Everyone would have their own opinions. It's evident that today's systems have no consciousness as such.

Figure 3 shows the quantitative and qualitative nature of the above listed fundamentals of intelligence and also a rough extent up to which they are being implemented in today's intelligent machines. 


\section{Fundamentals of Intelligence}

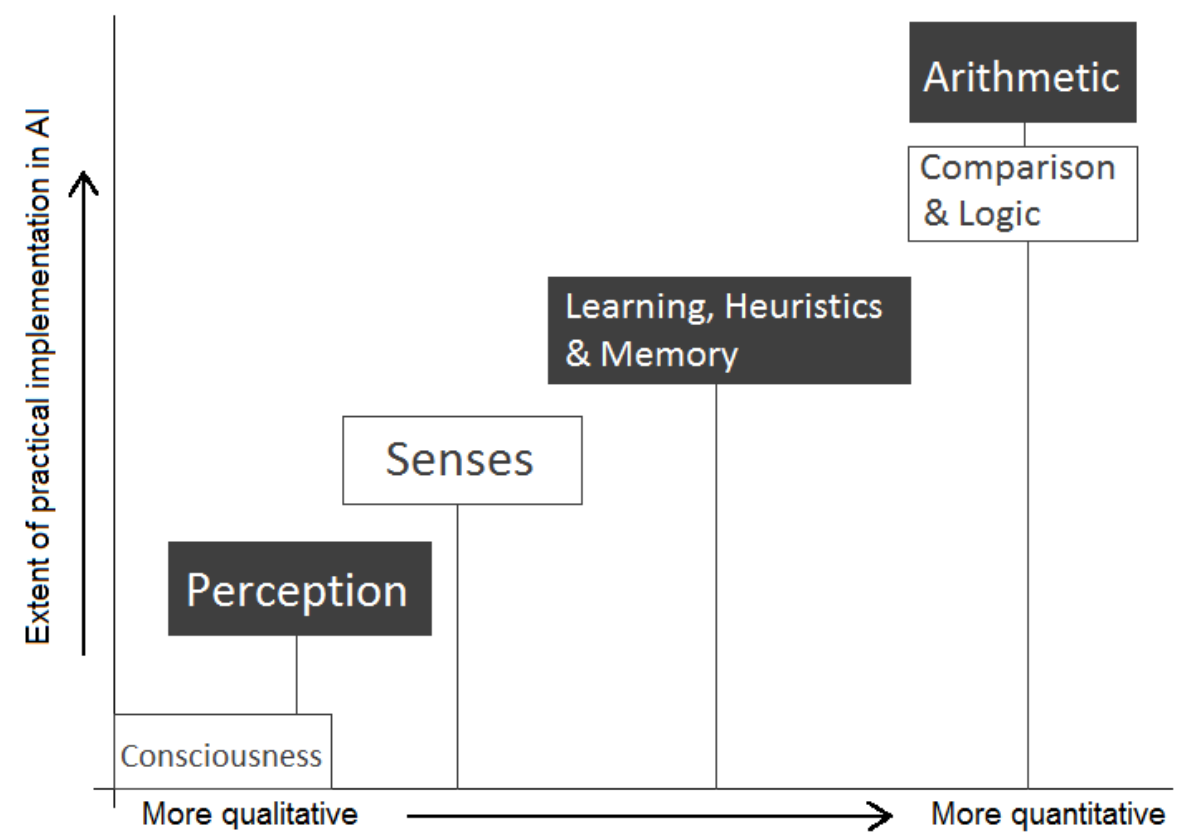

Figure 3. Analysis of Fundamental Traits of Intelligence and Today's Al

\section{Redefining Machine Intelligence}

This paper intends to offer a novel overview regarding Artificial Intelligence entities and systems. The statements given below in regard to a newly offered theory of machine intelligence provide the vision.

\subsection{Statement 1: Partially Intelligent Systems}

Any machine, system or program exhibiting some but not all of the above listed fundamentals of intelligence is a Partially Intelligent System. For example, chatbots exhibit some of above listed traits, namely Comparison, Logic \& Reasoning and Learning, Heuristics \& Memory. If there is an additional module, they may exhibit some more traits also, like ability to perform arithmetic operations. Therefore, chatbots are Partially Intelligent Systems. Moreover, today's machine intelligence is greatly confined to the boundaries of Partial Intelligence. Even highly capable robots of today are an instance of highly capable Partial Intelligence, as today's AI does not possess complete power of all the fundamentals of intelligence.

\subsection{Statement 2: Completely Intelligent Systems}

Any machine, system or program exhibiting all of the mentioned fundamentals of intelligence completely will be considered a Completely Intelligent System. Such an entity will represent the true power of AI.

\subsection{Statement 3: Performance Factor}

The Performance Factor of a system is a measure offered for representing the capability of an intelligent system in terms of the fundamental traits of intelligence it possesses. It is given by the number of apparent fundamental traits shown by the given system divided by the number fundamental traits of intelligence, i.e., 6. Its value ranges from 0 to 1 . Hence, today's chatbots showing two or three of the given traits get a 
Performance Factor score of 0.33 to 0.5. A "Completely Intelligent System" will have a Performance Factor of 1.

\section{Conclusion}

Artificial Intelligence is perhaps the most interesting as well as challenging field of research today. It has already proven itself for solving some major problems for mankind. In near future, AI will present itself on a larger canvas and will become integrated in our day-to-day lifestyle. But there is need to continuously look for new ideas for development and to make progress in already devised research. We will then look forward to solving such problems via intelligent systems, where human intelligence can err. The paper offered an alternate foundation theory of intelligence in machines. The basic work can be carried forward to develop advanced AI theories and systems. Moreover, there is utter requirement for new theories to emerge and develop, especially in a challenging field like Artificial Intelligence.

\section{Acknowledgements}

We pay sincere acknowledgment to all the authors of all the reference material. Also, the kindest blessings of our parents and all the teachers gave the zeal and faith to put forward this work. Thanks to them.

\section{References}

[1] B. A. Shawar and E. Atwell, "Chatbots: are they really useful?", LDV Forum, vol. 22, no. 1, (2007).

[2] A. M. Turing, "Computing Machinery and Intelligence", Mind, (1950), pp. 433-460.

[3] A. Khanna, "Pandorabots Chatbot Hosting Platform. SARANG Bot", (2015) April 19, Internet: http:// pandorabots.com/pandora/talk?botid=9f0f09a71e34dcf8/.

[4] A. I. Alice, "Foundation. Free A.L.I.C.E. AIML Set", (2015) March 21, Internet: http://code.google.com/p/aiml-en-us-foundation-alice/.

[5] B. Whitby, Author, "Artificial Intelligence", The Rosen Publishing Group, (2009).

\section{Authors}

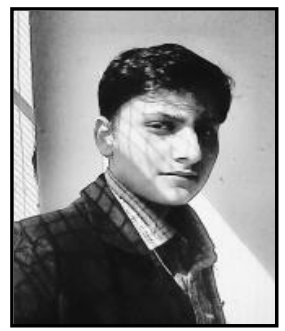

Anirudh Khanna, is a first year student of B.E. in Computer Science Engineering, at Chitkara University, Punjab. His interest areas are Artificial Intelligence, Programming, Web Development, Green Computing and etc. He is into the analysis and testing of algorithms, tools and techniques in Artificial Intelligence and development of novel theories for improvement.

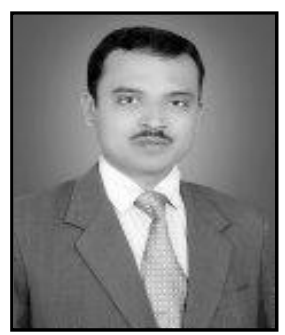

Bishwajeet Pandey, is asst. professor in Centre of Excellence of Chitkara University. He has worked as JRF at South Asian University and visiting lecturer in IGNOU. His research areas are Green Computing, Low Power VLSI, Energy Efficient Design, AI, Green Computing, etc. 


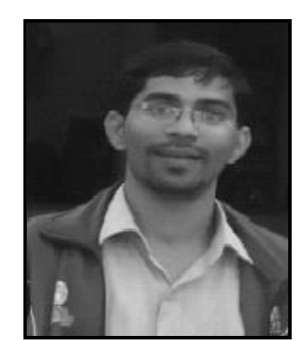

Bhale Pradeepkumar, has been working as asst. professor in CSE Department of Jawaharlal Nehru Engineering College, Aurangabad and has recently received Masters Degree (CSE) from IIITM, Gwalior. He has specialization in Information Security, A.I. and Data Mining.

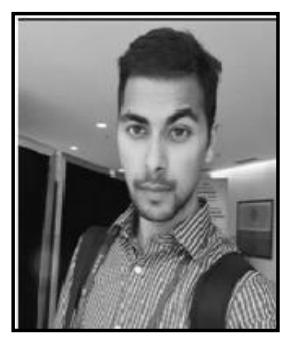

Kartik Kalia, is working as a researcher in Chitkara University Research and Innovation Network (CURIN). He is also appointed as a Director in Gyancity Research Lab. His area of interest is Low Power Research in Thermal Design for Energy Efficient Computing.

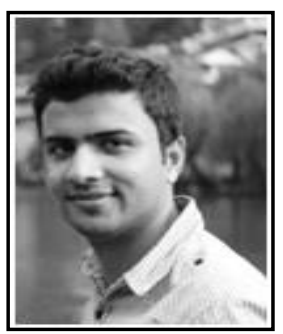

Teerath Das, is a doctoral student in Gran Sasoo Science Institute, Italy and has completed M.Sc. in Computer Science from South Asian University, New Delhi. His area of interest includes Low Power Techniques in VLSI and Digital Signal Processing. 